\title{
Gerencia estratégica sustentada por el costeo basado en actividades
}

\author{
Rodríguez Medina, Guillermo*
}

\section{Resumen}

En el presente trabajo se analizan los aspectos relevantes en que se sustenta la gerencia estratégica de costos, como la cadena de valores, el posicionamiento estratégico, las causales de costo, los costos de la calidad, las medidas no financieras de rendimiento en combinación con las financieras y el costeo basado en actividades, contrastándose con la gerencia convencional de costo. Se realizó una revisión bibliográfica que permitió sistematizar los aspectos conceptuales e identificar los factores claves de éxito del enfoque contemporáneo. Se hace énfasis en la importancia relativa de la carga fabril dentro de la estructura de costos de los productos o servicios en el actual contexto organizacional, caracterizado por la complejidad de los procesos, la exigencia de mayor intensidad de capital, la automatización y la multiplicidad de productos en diferentes niveles de producción. Esta situación no es captada por la gerencia tradicional y los informes generados inducen a la toma de decisiones erradas, mientras que la gerencia estratégica, a través del costeo basado en actividades permite determinar el costo con mayor exactitud de los productos o servicios. Su utilidad originalmente fue para mejorar los procesos manufactureros, pero en el devenir del tiempo se ha extendido a los diversos procesos de las diferentes organizaciones, por el énfasis que hace en las actividades que añaden valor para el cliente.

Palabras clave: Gerencia estratégica, cadena de valor, causales de costos, costo de la calidad, costeo basado en actividades.

\section{Recibido: 98-01-27, Aceptado: 99-02-17}

- Prof. de Costos en Pre y Postgrado de la Facultad de Ciencias Económicas y Sociales, Universidad del Zulia. Lic. en Contaduría Pública, Magister en Gerencia de Empresas, Mención Gerencia Industrial, candidato a Doctor en Ciencias, mención Gerencia. E-mail: Gprodrig@luz.Ve 


\section{Stategic Management Through Cost Analysis Based on Activities}

\section{Abstract}

This paper analyzes the relevant aspects that sustain strategic cost analysis, such as value chains, strategic positioning, the causes of costs, quality cost, financial and non-financial elements of productivity, and activity based costs, in contrast with conventional cost management. A bibliographical review permitted the systematization of the conceptual aspects and the identification of key factors in the success of the modern approaches. Emphasis is given to the relative importance of manufacturing costs within the cost structure for products and services in the present organization context, which is characterized by the complexity of the processes, the higher capital intensity levels, automation, and a multiplicity of products in different production levels. This situation is not covered by traditional management practices, and so the reports generated by them may lead to errors in decision making, while strategic management, through activity cost analysis, determines the service or product costs with greater precision. The original purpose of this analysis was to improve manufacturing processes, but with the passage of time it has been extended to diverse processes in different types of organizations, because of its emphasis on activities which ad value for the client.

Key words: Strategic management, value chains, cost causes, quality cost, activity based costing.

\section{Introducción}

La gerencia convencional de costos tiene su origen a finales del siglo pasado y se fundamenta principalmente en los aportes de Taylor, referentes al control de gestión industrial, con la finalidad de aumentar la productividad empresariai; para ello realizó los estudios de tiempo y movimiento de la mano de obra directa, a fin de incrementar la eficiencia del trabajador, y el control de las materias primas para disminuir el desperdicio de los materiales; pues, los materiales y la mano de obra eran los principales componentes de la estructura de costos de los productos y por tanto los elementos a controlar, los cuales representaban entre un $80 \%$ a un $90 \%$, los costos indirectos de fabricación constituían el porcentaje restante, y para cuya asignación se usaba generalmente como base el costo de los materiales directos, el costo de la mano de obra directa o el costo primo (Rodríguez y González, 1996:37). Pero hoy en dia las organizaciones industriales se desenvuelven en un mundo caracterizado por las rápidas y profundas transforma- 
ciones, demandadas por las presiones competitivas; la automatización, la depreciación y el mantenimiento de la tecnología, y la complejidad de las organizaciones le han dado una importancia relevante a los costos indirectos de fabricación; la mano de obra directa tiene ahora menos importancia en el valor agregado de los productos, convirtiéndose más bien en un elemento más de la carga fabril.

Esta situación creó bases suficientes para generar un cambio en la manera de administrar los costos de manufactura de las empresas, tanto desde el punto de vista cualitativo como cuantitativo, dando origen a la gerencia estratégica de costos de producción, sustentada principalmente por la conceptualización de la cadena de valores desarrollada por Porter desde 1987 (1997); por lo tanto este artículo intenta profundizar y aportar conocimiento sobre los elementos claves que se deben tener en cuenta para garantizar el éxito potencial de una gerencia estratégica de costos de manufactura, y la utilización del costo basado en actividades como instrumento que permite medir el costo de un producto o servicio de una manera más objetiva, porque rastrea el consumo de los recursos por las actividades, y la incidencia de estas en el costo de los productos o servicios, a través de una relación causal.

\section{Fundamentos de la Gerencia Estratégica de Costos}

En virtud de los constantes cambios que se originan en el ámbito organizacional, debido entre otras cosas a las innovaciones tecnológicas, producto de las presiones competitivas, que desbor- dan las fronteras nacionales para adentrarse en un mercado más amplio, las empresas se ven en la imperiosa necesidad de mejorar constantemente el valor de sus productos, mejorar su productividad y reducir sus costos.

La gestión de costos tradicional fundamentada, principalmente, en los aportes de Taylor, permitió el desarrollo de la contabilidad analítica, los estudios de tiempo y movimiento de la mano de obra directa, los estándares, la gestión de las existencias y de las materias primas, el premio al rendimiento y la asignación de los costos indirectos de fabricación con base en los costos de los materiales directos y la mano de obra directa con la finalidad de aumentar la productividad (Lorino, 1993:8).

Esta filosofía establecida desde finales del siglo pasado; deja mucho que desear en la actualidad, porque no capta, ni refleja la caracterización de las organizaciones de hoy en dia, como son la complejidad, la automatización, la multiplicidad de productos y el cambio permanente en los procesos y productos para penetrar y captar más mercado; esto ha originado un cambio de mentalidad en cuanto al análisis de los costos de producción, tanto desde el punto de vista cualitativo como cuantitativo, a partir de la conceptualización de la cadena de valor desarrollada desde 1987 por Porter (1997), la cual puede considerarse como la base fundamental en que se sustenta la gerencia estratégica de costos; ésta, según Shank y Govindarajan (1995:5) es aquella que analiza los costos dentro de un contexto más amplio, en el que los elementos estratégicos aparecen en forma más explícita, formal y consciente, y los datos de costos se utili- 
zan para desarrollar estrategias superiores, con el objeto de alcanzar ventajas competitivas sostenibles.

Según Shank y Govindarajan (1995:8) la gerencia estratégica de costos se fundamenta en una combinación equilibrada del análisis de la cadena de valor, del posicionamiento estratégico y de las causales de costo; pero considero además necesario agregar el análisis de los costos de la calidad y las medidas no financieras de rendimiento.

\subsection{Cadena de Valor}

La gerencia de costos convencional se fundamenta en el concepto de valor agregado; es un enfoque dirigido a los factores internos de la empresa y hace énfasis en los resultados financieros a corto plazo. Este enfoque convencional comienza con los pagos hechos a los proveedores (compras) y termina con los costos pagados por el consumidor (ventas); por lo tanto, desde el punto de vista estratégico, el concepto de valor agregado tiene dos problemas: comienza demasiado tarde y termina demasiado pronto (Shank y Govindarajan, 1995:16); es muy limitado, pues no analiza ni explota las relaciones con los proveedores de materias primas ni con los consumidores, que son la razón de ser de toda empresa.

En contraposición al enfoque tradicional tenemos el de la cadena de valor, herramienta que permite disgregar a la empresa en sus actividades estratégicas relevantes para comprender el comportamiento de los costos y las fuentes de diferenciación existentes y potenciales (Porter, 1997:51).
La cadena de valor de una organización, es solo una parte de la cadena de valor del sector en que opera, ya que ella debe estar íntimamente relacionada con los eslabones de la misma cadena de los proveedores, y con los de los canales de distribución hacia el comprador, que es denominado, por Porter, sistema de vaior. Comprender y examinar las interrelaciones de las actividades de la cadena de valor de una empresa, representa una fuente potencial de ventaja competitiva en la gestión estratégica de costos.

De acuerdo, a lo señalado por Porter (1997) la cadena de valor de una compañía está compuesta por nueve categorías de actividades genéricas que están relacionadas en forma particular: Cinco actividades primarias (logística interna, operaciones, logística externa, mercadotecnia y ventas, y servicio), que son las actividades implicadas en la creación física del producto, su venta y posterior servicio; y cuatro actividades de apoyo, (infraestructura de la empresa, recursos humanos, desarrollo tecnológico y abastecimiento), que sustentan a las actividades primarias y se apoyan entre sí.

Esta concepción de analizar los costos en un contexto más amplio y más exhaustivo, permite a la gerencia estratégica de costos tener una visión largo placista y desarroilar estrategias para obtener ventajas competitivas potencialmente sostenibles.

\subsection{Posicionamiento Estratégico}

El posicionamiento estratégico está referido a la manera como la empresa haya decidido competir, pues la esen- 
cia de la formulación de una estrategia competitiva consiste en relacionar a una empresa con su medio ambiente, sobre todo dentro del sector o sectores industriales en los cuales compite. Dado que las fuerzas externas por to general afectan a todas las empresas del sector industrial, la clave se encuentra en las distintas habilidades de las empresas para enfrentarse a ellas (Porter, 1980:23).

La situación de la competencia en un sector industrial está englobada en cinco fuerzas competitivas básicas: La entrada de nuevos competidores, la amenaza de sustitutos, el poder de negociación de los compradores, el poder de negociación de los proveedores y la rivalidad de los competidores existentes (Porter, 1997: 22). La acción conjunta de estas cinco fuerzas determina la rentabilidad potencial del sector industrial, en el sentido que el retorno sobre la inversión en el largo piazo debe ser superior al capital invertido.

La empresa debe conocer sus fortalezas, oportunidades, debilidades y amenazas, y de las causas de las fuerzas competitivas, lo cual le indicará pautas para determinar la estrategia competitiva, y decidir las áreas en que debe entrentarse a la competencia y en las que debe evitarla. Sobre este particular, Porter (1997:29) señala dos tipos básicos de ventajas competitivas que puede poseer una empresa: la de costos bajos y la de diferenciación de productos. Estos dos tipos básicos de ventajas competitivas, conllevan a establecer tres estrategias genéricas para lograr un desempeño superior al promedio en un sector industrial: liderazgo en costos, diferenciación y enfoque.
Los esquemas conceptuales en que se sustenta cada una de las estrategias genéricas son totalmente diferentes y por tanto requieren de diferentes perspectivas en el análisis de costos. La estrategia de liderazgo en costo se fundamenta en ofrecer a los consumidores a un menor costo, productos similares a los que ofrecen los competidores; la estrategia de diferenciación se sustenta en ofrecer productos con uno o más atributos que los clientes perciben como importantes y exclusivos; $y$ la estrategia de enfoque descansa en la elección de un panorama de competencia estrecho, especifi$\mathrm{co}$, dentro de un sector industrial, pues, selecciona un grupo o segmento del sector industrial, y desarrolla su estrategia a partir de esta concepción. Esta última estrategia, según Porter (1997:33), tiene dos variantes: el enfoque de costo que explota las diferencias en el comportamiento de costos en algunos segmentos, y el enfoque de diferenciación que explota las necesidades especiales de los compradores en ciertos segmentos.

Las estrategias de liderazgo en costo y diferenciación buscan la ventaja competitiva en un mercado amplio, mientras que la estrategia de enfoque la busca en un mercado meta. Esta última estrategia, de acuerdo a Thompson y Strickland (1994:124) es atractiva cuando se cumplen las siguientes condiciones:

- El segmento es suficientemente grande para ser rentable.

- El segmento tiene un buen potencial de crecimiento.

- El segmento no es crucial para el éxito de los competidores importantes. 
- La compañía tiene las capacidades y los recursos para satisfacer al segmento de manera eficaz.

- El especialista se puede defender de los desafíos basado en el buen nombre que ha creado entre sus clientes y en su capacidad superior de satisfacer a los competidores del segmento.

La estrategia es el medio para obtener la ventaja competitiva y una vez obtenida surge el liderazgo dentro del sector industrial, no como una causa sino como una consecuencia de la ventaja competitiva; obtenido el posicionamiento del mercado la empresa no solo está en capacidad de reaccionar frente a su medio, sino de influir en él.

Sin embargo, considero que independientemente de la estrategia que se establezca, los costos juegan un papel importante en la posición estratégica de una compañía en relación a sus competidores, porque incluso la que utiliza la de diferenciación, cuyo objetivo es resaltar las cualidades particulares de sus productos, debe hacerle un seguimiento a sus costos para tenerlos a tono con su proceso y evitar cualquier peligro de la competencia.

\subsection{Causales de Costos}

En cuanto al análisis de las causales de costos, la gerencia estratégica reconoce que los costos son causados o impulsados por múltiples factores que se hallan interrelacionados en forma compleja. La comprensión del comportamiento de los costos implica conocer la interrelación del conjunto de conductores, im- pulsadores o factores de costos que se toman en cuenta cuando se desagregan los costos de los recursos o de las actividades.

Para un mejor discernimiento de las causales de costos es conveniente contrastar la gerencia convencional con la estratégica; en la primera se considera como principal causal de costo el volumen de producción, sobre el cual se fundamenta el comportamiento de los costos; mientras que la segunda considera que el volumen de producción capta muy poco sobre la riqueza del comportamiento de los costos, y por el contrario trabaja con muchas causales de costos, que pueden ser divididas en estructurales y de ejecución. Las causales estructurales de costo se fundamentan en la estructura económica de la organización; existen al menos cinco opciones estratégicas, que permiten la obtención de un costo competitivo para cualquier grupo de productos (Shank y Govindarajan, 1995:26):

Escala: cuantia de la inversión a efectuar en producción, marketing e investigación y desarrollo.

Extensión: Grado de integración vertical. La integración horizontal está más relacionada con la escala.

Experiencia: Las veces que en el pasado la empresa ha realizado lo que está haciendo ahora.

Tecnología: Métodos tecnológicos utilizados en cada etapa de la cadena de valor de la empresa.

Complejidad: Amplitud de la línea de productos o servicios a fin de ofrecer opciones variadas a los clientes. 
Las causales de costo de ejecución, son aquellas que reflejan la habilidad de la empresa para el establecimiento y mejoramiento de la posición de costo adoptada, y están sometidas a métricas de rendimiento y desempeño, mientras que las causales estructurales no. Además, el hecho de haber más causales de costo de ejecución siempre es mejor, mientras en las causales estructurales de costo no sucede asi, pues, una línea de producto más compleja no es necesariamente mejor o peor que una línea menos compleja y demasiada experiencia puede ser tan nociva como muy poca experiencia en un ambiente dinámico (Shank y Govindarajan, 1995:27).

Las causales de costo de ejecución tienden a ser más utilizadas que las estructurales en los análisis estratégicos de costo, debido a que permiten mejorar los procesos productivos de la cadena de valor. Entre las causales de costo de ejecución se incluyen al menos las siguientes (Mallo y Merlo, 1995:389):

Implicación de la fuerza de trabajo mediante la participación. Compromiso de la fuerza de trabajo para una mejora contínua.

Gestión de la calidad total. Creencias y realizaciones en relación con la calidad de productos y procesos.

Utilización de la capacidad, Dada la escala elegida.

Grado de eficiencia. En relación con las normas corrientes, de la disposición en planta.

Configuración del producto.
Explotación de las relaciones con suministradores y/o clientes. Para la cadena de valor de la empresa.

Para un análisis estratégico de costo el volumen de producción no es la mejor manera para analizar el comportamiento de los costos; este se debe realizar a través de las causales de costos, ya sean estructurales o de ejecución, pero haciendo énfasis en éstas últimas, porque permiten medir el desempeño. El examen de las causales de costo facilita entender el comportamiento del costo en cada actividad de la cadena de valores. por lo cual el costeo basado en actividades, se convierte en un instrumento útil para hallar una relación causa-efecto en dicho comportamiento. A este tema dedicaremos la segunda sección del presente artículo.

\subsection{Costo de la Calidad}

Además de los temas tratados anteriormente, la gerencia estratégica de costos debe considerar la gestión de calidad, tanto desde el punto de vista cualitativo como cuantitativo; la administración de la calidad es un elemento fundamental en el juego de la competitividad empresarial, porque persigue la mejora continua de los procesos; tanto es así, que los países pioneros en la gestión de calidad total, como Japón, todavía siguen a la vanguardia con esta filosofía.

La calidad es una medida relativa de lo bueno de un producto o servicio, pero el último juez de la calidad es el cliente; por lo 
tanto, un producto o servicio de calidad es el que alcanza o excede las expectativas del cliente (Hansen y Mowen, 1996:439). Por otro lado, Alvarez y otros (1996:284) expresan que la calidad consiste esencialmente en producir al menor costo posible productos o servicios que satisfagan las necesidades de los clientes y a la vez ofrecer la máxima motivación y satisfacción a los empleados.

Como puede observarse, este concepto de calidad coloca en primer lugar la reducción del costo y en segundo término la satisfacción del cliente respecto al producto. Asume implícitamente que el primer elemento que induce al cliente a comprar es el precio. pero considero, por un lado, que el valor, es decir, la utilidad del producto, es el factor para ser demandado por los consumidores, en concordancia con el precio, y por el otro lado para que las especificidades del producto se cumplan, el personal que labora en él debe estar identificado con el proceso, motivado y satisfecho en su ego.

La calidad actualmente se considera un factor clave de competitividad y las empresas que implantan esta filosofía buscan la mejora constante de sus procesos y productos, tratando de disminuir costos, para satisfacer tanto las expectativas de los clientes como la motivación del personal que labora en ella.

La calidad de cualquier producto o servicio depende de la interacción de la calidad de diseño, la calidad de la conformidad con el diseño y la calidad del desempeño (Polimeni y otros, 1994:404). La calidad del diseño se refiere a las especificaciones del producto o servicio, la calidad de conformidad consiste en la fabri- cación del producto o servicio de acuerdo con las especificaciones señaladas en el diseño y la calidad del desempeño depende de la dos anteriores, lo cual significa que si la calidad del diseño o la conformidad no cumplen con las exigencias de una buena calidad, el producto o servicio ofrecerá un pobre desempeño, es decir, se ofertará un bien o servicio de baja o mala calidad, lo que implica incurrir en costos financieros adicionales, como son los retrabajos o reparaciones. Además es necesario establecer indicadores no financieros, que permitan mejorar los procesos, tales como el número de quejas de los clientes, el porcentaje de unidades defectuosas enviadas a clientes, etc.

Los costos de calidad se refieren a los costos incurridos para evitar que haya calidad pobre o aquellos costos realizados porque ya hubo una mala calidad (Horngren y otros, 1996:795).

Cuando los programas de calidad forman parte de la evaluación de gestión, los costos asociados con la calidad pueden generar ahorros considerables de costos y aumentar los ingresos. Estos costos están relacionados con la identificación, reparación y prevención de defectos.

Los costos de calidad se clasifican en cuatro categorías (Horngren y otros, 1996:795)

Costos de prevención: Son los que se realizan para evitar la fabricación de productos que se desvien de las especificaciones.

Costos de evaluación: Son los incurridos para detectar cuál de las unidades individuales no se apega a las especificaciones. 
Costos de falla interna: Son aquellos costos cuando se detecta que un producto no se apega a las normas, antes de enviarse al cliente.

Costos de falla externa: Son los que se incurren cuando se detecta que un producto no se apega a las normas, después de enviarse al cliente.

De los costos anteriores, los costos de prevención y evaluación, pueden incrementarse porque son necesarios para obtener la buena calidad, mientras que los costos de fallas internas y externas deben tender a su eliminación porque son costos ocasionados por la no conformidad, o mala calidad. De acuerdo a Hansen y Mowen (1996:438) los costos de calidad para las empresas estadounideses ascienden generaimente de 20 a $30 \%$ de las ventas; $y$ los expertos en calidad sostienen que el nivel de calidad óptimo debe ser un $2,5 \%$ de las ventas. Por otro lado Cornejo (1992) afirma que un producto de calidad trae siete clientes potenciales mientras que uno de mala calidad aleja a veintidós clientes potenciales. De tal manera que las organizaciones deben comprender que la razón de ser de su existencia son los clientes, y la satisfacción de éstos debe ser la prioridad número uno, pues un producto de buena calidad sólo es redituable sí también satisface a los consumidores, porque tiende a reducir costos e incrementar los ingresos a través del aumento de la demanda.

Por otro lado hay que tener en cuenta los costos en que se incurre cuando no se trabaja con calidad, porque según Blanco ( $\$ / f: 525$ ) conducen a una pérdida de competitividad y entre ellos tene- mos los costos de las ventas perdidas, (disminución de beneficios y erosión de la cuota de mercado), los costos de insatisfacción del cliente, los costos en que éste incurre y los costos de la mala calidad en la gestión. Por tanto la consideración de los costos de la calidad y la no calidad son factores claves para el éxito gerencial estratégico de costos.

\subsection{Medidas no financieras de rendimiento}

De acuerdo a Horngren $y$ otros (1996) las medidas de rendimiento son componentes centrales de un sistema de control de gestión, que pueden ser financieras o no.

La gerencia estratégica de costos considera, además de las medidas financieras en que se sustenta la gerencia de costos convencional, las medidas no financieras de rendimiento como instrumento de medición, considerándose otro factor clave de éxito al igual que las causales de costo, la calidad del producto, la productividad, el tiempo, las satisfacciones de los clientes y de los empleados entre otros. Las medidas financieras, son los criterios de medición más usados por la gerencia de costos tradicional; proporcionan generalmente información sobre el desempeño de la eficacia financiera en un período dado, tanto desde el punto de vista de la contabilidad administrativa, donde los informes generados son para fines internos de la organización, como de la contabilidad financiera cuyos informes pueden ser utilizados tanto para fines internos como externos, y están sustentados sobre hechos pasados. 
La gerencia estratégica de costos, además de las medidas no financieras, considera las financieras, porque las primeras dirigen la atención a los procesos físicos y marcan pautas para reflejar las debilidades de los procesos operativos, permitiendo las correcciones pertinentes y oportunas en la ejecución de los procesos, facilitando la búsqueda de la mejora continua, la supervivencia y el crecimiento de la organización, en un mundo convulsionado por la competitividad y la globalización, mientras que las medidas financieras permiten cuantificar el control de los costos y determinar el nivel de rentabilidad.

Las medidas no financieras de desempeño sirven como instrumento básico de control estratégico; los resultados obtenidos por su utilización, permiten la comparación con la competencia, siendo el benchmarking, una herramienta para tal fin. Al respecto Mallo y Merlo (1995:65) definen al benchmarking como el proceso continuo de medida de los productos, servicios y procesos en relación con los competidores más fuertes o aquellos considerados como líderes mundiales en sus sectores.

Las medidas no financieras de desempeño tienen una importancia relevante al utilizarlas en factores como las causales de costo, servicios a los clientes, satisfacción de los empleados, productividad y tiempo; que no están basados en unidades monetarias, pero tienen que usarse en combinación con las medidas financieras, porque según Alvarez $y$ otros (1996:332) existen medidas no financieras tales como número de ajustes, tasas de defectos, tiempo de ajuste, utilización de máquinas, calidad y satisfacción al cliente, que pueden tener un claro componente de subjetividad, el cual dificulta la verificación por parte de un auditor, así como podrian tener distintas aplicaciones e incluso interpretaciones.

Las medidas no financieras tienen que usarse en forma mancomunada con las financieras en una combinación óptima en cada nivel de responsabilidad en aras del objetivo de la organización, ya que tienen efectos positivos en los resultados operativos y por ende en los financieros.

\section{El Costeo Basado en Actividades (C.B.A.)}

El costeo basado en actividades (C.B.A.), de acuerdo a Brinson (1997) y a Horngren y otros (1996), es un método cuya característica distintiva es su enfoque en las actividades, donde éstas consumen recursos (humanos, materiales y de inversión), mientras que los objetos de costos (productos, servicios y clientes) consumen actividades. Además, Amerio y otros (1998) quienes citan a Castillo, Howell y Soucy, agregan que las actividades se combinan en cadenas o en redes de actividades llamadas procesos, destinadas al logro de un objetivo global, es decir, a una salida global material o inmaterial, lo que implica que el costo de cada proceso incluye la sumatoria de los costos de las actividades que lo conforman.

Este tipo de costeo es una herramienta utilizada por la gerencia estratégica de costos, porque permite a las empresas adaptarse con facilidad a los cambios dinámicos de las organizaciones en cuanto al avance tecnológico, a la complejidad del proceso productivo, a la di- 
vensidad de la producción, entre otros, y permite determinar el costo del producto o servicio (objeto de costo) de una manera más exacta que el costeo convencionat porque en el C.B.A., lo fundamental es determinar el costo de las actividades, lasicuales consumen diferentes recursos, $y \in$ s costo del producto o servicio es una consecuencia de las actividades.

El sistema de C.B.A. puede ser utilizído en el costeo por órdenes especificas, en el costeo por proceso o cualquier otro tipo de costeo, y tiene la ventaja de determinar el costo unitario con mayor exactitud que el sistema tradicional; proporciona a la administración información méss pertinente para la toma de decisiones y hace énfasis en el control administraiivo de las actividades, tanto desde el punto de vista de costo como para mejorar la eficacia de las operaciones; el
C.B.A. involucra a las personas que laboran en el proceso productivo para que se hagan responsables por las actividades que realizan, tratando siempre de proporcionar soluciones a los problemas que se les puedan presentar en el proceso, tales como los derivados del tiempo de ajuste de la maquinaria, el número de averías y otras medidas de carácter no financiero, con el objeto de controlar y mejorar su eficiencia.

Bajo este marco conceptual, lo relevante en el C.B.A., es determinar las actividades que agregan valor y reducir o eliminar las actividades estériles, que sólo incrementan el costo de los productos, servicios y clientes.

Una actividad, según Alvarez y otros (1996:308), quien cita a Castello y Lizcano, "es un conjunto de actuaciones o de tareas que tienen como objetivo la atri-

\section{Estructura del costeo basado en actividades}

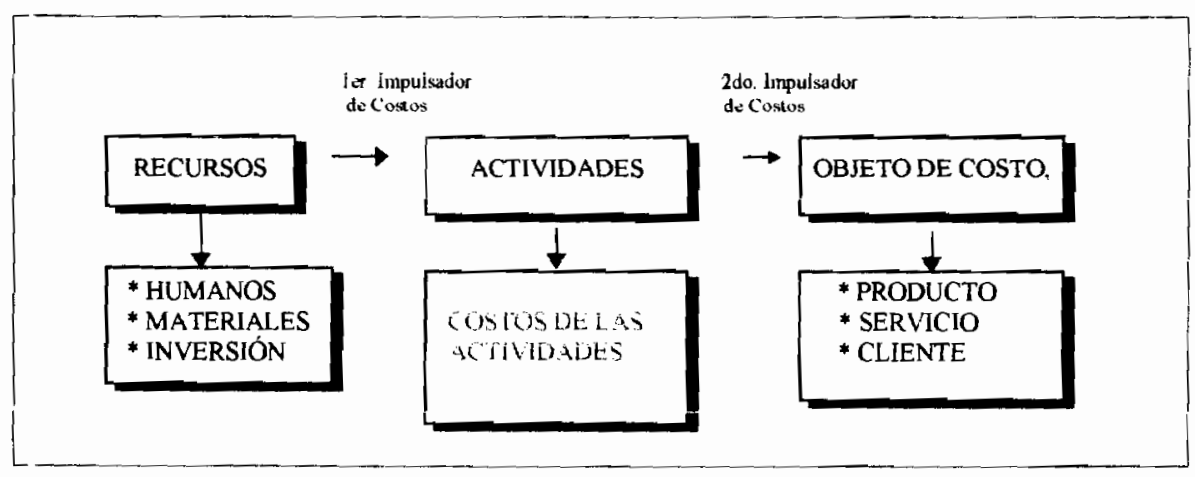

Fuente: Elaboración propia. 
bución, al menos a corto plazo, de un valor añadido a un objeto (producto o proceso), y permitir añadir este valor, bajo la perspectiva del cliente o usuario del mismo". Las actividad se caracteriza por ciertos atributos, entre los cuales se tienen:

a. Es repetitiva, en cuanto al proceso que desarrolla.

b. Consume recursos de costo y tiempo.

c. Tiende a obtener un output (producto, servicio, cliente).

d. Está condicionada por variables tales como: tiempos limitados, cumplimiento de calidad, frecuencia, etc.

e. Una actividad en un determinado contexto, puede ser una simple tarea en otro contexto o empresa.

Además de los atributos de las actividades hay que tener en cuenta que éstas se clasifican de múltiples maneras y cada organización las clasificará de acuerdo a sus necesidades particulares. Según Alvarez y otros (1996:310) las actividades se pueden clasificar de la siguiente manera:

- Por funciones: Compras, producción, ventas.

- Por su naturaleza: Actividades fundamentales $y$ actividades discrecionales.

- Por su relación con el objeto de costo: Actividades derivadas del volumen de producción, actividades relacionadas con la organización de los procesos productivos, actividades de sostenimiento de un producto, actividades de infraestructura.

- Por su aportación a la generación de valor: Actividades con valor agregado y actividades $\sin$ valor añadido.
Las actividades son, pues, las tareas específicas en que se descomponen los procesos operativos de toda organización; el análisis de ellas permite identificar las actividades relevantes y las despilfarradoras de costos, con la finalidad de hacer los correctivos necesarios en la búsqueda del mejoramiento continuo de los procesos operativos y del rendimiento. Según Brinson (1997:96) la mejora continua tiene varios objetivos:

- Eliminación de las actividades que no añaden valor.

- Mejora del rendimiento de las actividades que añaden valor.

- Sincronización del tiempo de espera en la introducción de nuevos productos y en el ciclo de producción.

- Mejora de la calidad.

- Eliminación de las desviaciones en los procesos corrigiendo la fuente de la desviación.

- Simplificación de las actividades.

El análisis de las actividades permite a la gerencia basada en actividades, la utilización de los recursos de la mejor manera posible para alcanzar los objetivos propuestos.

La importancia del C.B.A. radica en que determina el costo unitario del producto con una mayor exactitud que en el sistema tradicional, porque en éste último cuando se fabrican varios productos a diferentes niveles $y$ el proceso productivo es complejo y automatizado, se origina un subsidio cruzado de los que se elaboran en mayor volumen hacia los de menor cantidad de producción, determinando un costo divorciado de la realidad, cuestión 
que no sucede en el C.B.A.; éste último genera información vital y pertinente para que la gerencia mantenga a la empresa en el marco del entorno competitivo de nuestros días.

El subsidio cruzado de costo originado en el costeo tradicional, se debe principalmente a que los costos indirectos de fabricación, se consumen en diferentes proporciones a cada producto, situación que no es percibida por el costeo convencional, y lo que generalmente hace es asignar dichos costos a los productos, a través de medidas financieras relacionadas con el volumen de producción; pero realmente hay actividades que tienen que ver muy poco con el volumen, siendo lo más conveniente asignar los costos de esas actividades a través de una relación causal, que generalmente son medidas no financieras, como es el caso del costo de la actividad de despacho, que debería ser asignado de acuerdo al número de despacho.

Actualmente las innovaciones tecnológicas y las intensificaciones de capital, en los procesos de fabricación automatizados y la complejidad de los mismos, ha hecho que se haya invertido la estructura de costo de los productos o servicios; anteriormente (costeo tradicional) lo que interesaba rastrear eran los costos primos, es decir, los materiales directos y en especial la mano de obra directa, por ser los procesos de caracteristicas manuales, que representaban entre un 80 y un $90 \%$ del costo total, y el porcentaje restante correspondía a los costos indirectos de fabricación (Rodríguez y González, 1996), a los cuales no valía la pena hacerles un seguimiento porque no pasaban la prueba costo - beneficio; mientras que en el contexto presente (costeo contemporáneo), por las situaciones antes mencionada, los costos indirectos de fabricación tienen una importancia relevante, que según Echenique (1997) representan aproximadamente un $60 \%$ del costo total, mientras que la mano de obra directa ha descendido a un $15 \%$ de dicho costo, por la automatización de los procesos. Esta situación ha hecho que se cambie el paradigma de costeo, surgiendo como aiternativa el C.B.A. porque de lo contrario, si se sigue utilizando el costeo tradicional, la información generada puede inducir a decisiones gerenciales erróneas.

Un sistema de costeo basado en el volumen, donde los costos indirectos de fabricación se asignan con base a los costos de mano de obra directa, puede conducir a que los gerentes piensen que al bajar los costos de mano de obra directa también bajan los costos referentes a la carga fabril, lo cual es falso. Por ello la gerencia estratégica de costos hace énfasis en las medidas no financieras, para la búsqueda de las mejoras operacionales, sin descuidar la utilización de las medidas financieras, que permitan marcar pautas de comparación de la empresa con relación a los competidores.

\section{Conclusiones}

La gerencia estratégica de costo ha cambiado el paradigma de registrar y acumular los costos de la contabilidad convencional, debida a que las innovaciones tecnológicas, la complejidad de los procesos, la automatización de los mismos y la multiciplidad de productos en diferentes volúmenes de producción y el 
ciclo de vida de ellos, han hecho que la estructura de costo de los productos o servicios, se haya invertido. Especificamente, esto es así en lo referente a la mano de obra directa y a los costos indirectos de fabricación, pues en la contabilidad tradicional la mano de obra directa representaba un alto porcentaje de la estructura de costo debido a la caracterización de los procesos manufactureros de labor manual intensiva, mientras que el porcentaje de los costos indirectos de fabricación era insignificante con respecto a los materiales directos y la mano de obra directa.

Actualmente, los costos indirectos de fabricación tienen una importancia relevante en contraposición con la mano de obra directa que ha pasado a constituir un porcentaje insignificante y de acuerdo a autores como Shank y Govindarajan (1995), y Horngren et al (1996), un número creciente de organizaciones la considera formando parte del costo indirecto de fabricación.

Esta situación ha permitido el surgimiento del costeo basado en actividades como sistema para registrary acumular el costo de los productos o servicios, el cual permite rastrear los costos indirectos de fabricación a través de las actividades, utilizando como factores de costos frecuentemente variables no financieras que tengan relaciones causales para sus asignaciones.

Esta manera de costear los productos o servicios ha permitido el surgimiento de la Gerencia Basada en Actividades (G.B.A.), que de acuerdo a Horngren y
Sundem (1994:131), quienes citan a Peter Turney, utiliza la información generada por el costeo basado en actividades para tomar mejores decisiones; y está enfocada a mejorar el valor recibido por el cliente, además de mejorar las utilidades de la organización al proporcionar dicho valor, pues, hace énfasis en que las actividades de administración que sean necesarias se lleven a cabo de manera eficiente y reducir o eliminar las que no agregan valor.

Por otro lado tenemos que la implantación de un sistema de costos basado en actividades es complejo y oneroso, y no todas las compañías están dispuestas a utilizarlo, sobre todo aquellas organizaciones que todavía mantienen una estructura organizativa tradicional, por lo que resulta cuesta arriba pasar la prueba costo beneficio.

Por último consideramos la conveniencia de aprovechar la coyuntura del cambio curricular de estudio de la carrera de Contaduría Pública de la Escuela de Administración y Contaduria Pública de la Universidad del Zulia, para fomentar e introducir cambios sustanciales, en las cátedras relacionadas con estos tópicos, como son las de costos y administración, referentes al manejo de esta herramienta de carácter gerencial, en pro de una excelente formación académica de los egresados, de modo que puedan sumarse a la sociedad del conocimiento vanguardista y garantizar el éxito potencial en un mercado laboral caracterizado por la competitividad desde las diferentes aristas del saber. 


\section{Bibliografía citada}

Alvarez L, José y otros (1995). Contabilidad de Gestión Avanzada. Planificación, control y experiencias prácticas. Mc Graw-Hill, España.

Amerio, Ana; Viloria, Orlando; De Freites, Vidalina y Nava, Luis (1998). Asignación de Costos por Actividades para la pequeña y Mediana Industria del Calzado. Rev. Venezolana de Gerencia, año 3 № 6.

Blanco, Felipe. Contabilidad de costes y analítica de gestión para las decisiones estratégicas. 2da edición ampliada. Ediciones Dehusto S.A. España.

Brimson, James (1997). Contabilidad por Actividades. Aliaomega Marcombo. México.

Cornejo, Miguel Ángel (1992). Excelencia Directiva para Lograr Competitividad. Video conierencia. Lima, Perú.

Echenique, Luis (1997). El Costo basado en las actividades. Conferencia en el Colegio de Contadores Públicos del Estado Zulia, V Jornada de Actualización, Maracaibo - Venezuela.

Hansen, Don y Mowen, Mryanne (1996). Administración de costos. Contabilidad y control. Internacional Thomson editores, S.A. de C.V., México.

Horngren, Charles; Foster, George; Datar, Srikant (1996). Contabilidad de Costos. Un enfoque gerencial. Octava edición. Prentice Hall Hispanoamericana S.A., México.

Horngren, Charles y Sundem, Gary (1994). Contabilidad Administrativa. Nove- na edición. Prentice Hall Hispanoamericana S.A. México.

Lorino, Philippe (1993). El control de gestión estratégico. La gestión por actividades. Alfaomega. Marcombo, España.

Mallo, Carlos y Merlo, José (1995). Control de gestión y control presupuestario. Mc Graw-Hill, España.

Polimeni, Ralph; Fabozzi, Frank y Adelberg, Arthur (1994). Contabilidad de costos. Conceptos y aplicaciones para la toma de decisiones. McGraw-hill, Colombia.

Porter, Michael (1980). Estrategia competitiva. Técnicas para el Análisis de los Sectores Industriales y de la Competencia. Compañía editorial Continental S.A., México.

Porter, Michael (1997). Ventaja competitiva. Creación y sostenimiento de un desempeño superior. Editorial continental, México.

Rodríguez, Guillermo y González, Alitza (1996). Costos del servicio de cirugía ambulatoria basados en actividades. Caso Hospital Universitario de Maracaibo. La Universidad del Zulia. Facultad de Ciencias Económicas y Sociales. Trabajo de grado para optar al titulo de Magister en Gerencia de Empresas, mención Gerencia Industria!. Maracaibo.

Shank, John K; Govindarajan, Vijay (1995). Gerencia estratégica de costos. La nueva herramienta para desarrollar una ventaja competitiva. Grupo editoriai Norma, Colombia.

Thompson, Arthur y STRICKLAND, A.J. (1994). Dirección y Administración Estratégicas. Conceptos, casos y lecturas. Addison - Wesley Iberoamericana. Estados Unidos Americanos. 